\title{
Towards an analytics and an ethics of expertise: learning from decision-aiding experiences in public risk assessment and risk management
}

\author{
Myriam Merad • Nicolas Dechy • Michel Llory• \\ Frédéric Marcel $\cdot$ Alexis Tsoukias
}

Received: 27 September 2012/ Accepted: 19 December 2013/Published online: 15 January 2014 (C) Springer-Verlag Berlin Heidelberg and EURO - The Association of European Operational Research Societies 2014

\begin{abstract}
Public expertise in safety, security and environment (SSE) is a process that is increasingly submitted to control and transparency. As decision-making, the exercise of expertise involves subjectivity and judgment. An oversight, a monitoring and an aiding approach is therefore required for its conduct and its governance. This paper proposes a novel way of embedding ethical aspects and participative decisionmaking elements into the process of risk assessment and risks management. Based on their experience feedback, the authors first propose some early contributions to study the validity and the legitimacy of expertise in SSE. In the second part of the paper, the authors give an overview on how public expertise is organized in France and how the problems and the conclusions are framed in SSE. Finally, the authors propose a generic integrated framework for public expertise that constitutes the "responsible exercise of expertise". This framework allows framing a valid and a legitimate expertise process and its conclusions.
\end{abstract}

Keywords Expertise - Risks · Analytics · Ethics - Safety - Security and environment

Mathematics Subject Classification 00 General

M. Merad $(\bowtie) \cdot$ F. Marcel

INERIS, Parc technologique ALATA, BP2, 60550 Verneuil en Halatte, France

e-mail: merad.myriam@yahoo.fr; myriam.merad@ineris.fr

N. Dechy

IRSN, BP 17, 92262 Fontenay-aux-Roses Cedex, France

M. Llory

IAO Conseil, Bompas, France

A. Tsoukias

Université Paris Dauphine, Place du maréchal de Lattre de Tassigny, Paris, France 


\section{Introduction}

Major technological disasters and accidents around the world; for example, Minamata in the early 1950s and 1960s or more recently AZF in Toulouse (France) in 2001 or Fukushima in 2011, regulation such as Aarhus convention in 1998, controversies and societal mobilizations have contributed to the discussion of the limits of public decision-making in safety, security and environment (SSE). Public expertise, as a decision-aiding process and as a part of the decision-making process in SSE, is nowadays questioned by public opinion and subject to doubts and criticisms.

Decision-aiding process has already been discussed by Roy (1993), Bouyssou et al. (2000, 2006) and Tsoukiàs (2007). A decision-aiding process implies the existence of at least a Client (that can represent the DM and other actors) and an Analyst. This process makes sense with respect to one or more decision processes (Tsoukiàs 2007). SSE decision processes are mainly public because they deal with "public objects or goods" with respect to Ostrom's (2005) definition: goods or objects with low subtractability of use and a high difficulty of excluding potential beneficiaries. These objects are of interest for different actors that are for some of them public actors (e.g., Ministry, local administrations...) (Ostanello and Tsoukiàs 1993). These public actors are, in a large majority of situations, responsible and accountable for the decisions that will be taken in SSE.

Expertise in SSE is a public decision-aiding process according to the above definition. The complexity of situations in SSE, the intrinsic uncertainty on the public objects and the variability of interaction situations between the Expert, the Client, the DM and the other actors make the process of Expertise and the impact of expertise conclusions difficult to predict. That is why the Expertise process in SSE is also a "Public decision process" that needs a decision-aiding process. In this case, the Client can be a set of experts and the Analyst can be a member of the expertise team. The Analyst must excel in methods in the conduct and the governance ${ }^{1}$ of expertise and on one or different domain of SSE.

In Merad (2010), we discussed the concept of public expertise as a link between the scientific and the political worlds. For some people, public expertise in SSE is limited to the mastering of scientific knowledge without being influenced by the ongoing regulations, socio-economical, political and technical contexts. The objective is to provide a technical and scientific expertise that is strictly independent from the decision process. For some others, the expertise is an art based on experiences and intuitions, which can only be recognized by peers. In that sense, it is extremely difficult to describe the underlying mechanism of expertise. For others, the expertise in SSE is the development of know-how in the application of the standards and rules in practice. For some others, expertise is a function defined by the administration (in the sense that if a member of a public expertise agency is performing an expertise then he is an expert) and not a characteristic (someone is

\footnotetext{
${ }^{1}$ Governance refers to the actions, processes, traditions and institutions by which authority is exercised and decisions are taken and implemented (see "An introduction to the IRGC- Risk GovernanceFramework" published by the IRGC in 2008).
} 
involved in a public expertise because of his methodological and/or scientific knowledge and experience $)^{2}$. So to speak, public expertise depends on specific endogenous characteristics, such as for example neutrality, competence, the ability to negotiate the capacities of communication, and on exogenous characteristics such as, for example, the function, the mandate and the peer recognition. The reader can complete this discussion in Favro (2009) and Merad (2010).

In this paper, we argue that public expertise needs a decision aiding for experts. We then propose an integrated framework for analytics and ethics of expertise in its conduct and its governance. Conducting a public expertise in SSE consists in describing the context of expertise, in formulating and modeling the expertise issues and problems and in framing consistent conclusions. Governance of public expertise in SSE refers to decision-making, actions, processes, regulation, traditions, organizations and institutions that characterize the way expertise is organized and regulated and to how SSE conclusions are taken into account, accepted, negotiated with stakeholders and implemented in SSE decision processes (or the SSE decision process). This integrated framework is submitted to two main conditions: validity and legitimacy.

In Section 1, we will describe some open questions about legitimacy and validity of expertise in SSE. Initial answers will be provided, the state-of-the-art will be discussed and an integrated framework for analytics and ethics of expertise will be introduced. Section 2 will be dedicated to defining more precisely public expertise and to explicating and discussing main concepts. Section 3 will be dedicated to precising the way the integrated framework is structured based on engineering of decision-aiding process to support expertise in SSE experience feedback.

\section{Early contributions to study the validity and the legitimacy of expertise in SSE}

\subsection{Learning from experience: open questions and first answers}

Given a long experience working on the field of risk prevention within the public domain on natural and on technological hazards, we have always been facing along the road what some will consider as being "ethical considerations". We have then faced questions such as "Expertise is separated from Decision: Why do you feel so concerned by the potentials outcomes of your recommendation?" and "What can you possibly learn and give as added values by investigating the decision and governance context and re-questioning the usual expertise methods?".

Nowadays, these ethical considerations are numerous and follow the continual sliding between what is a science conclusion and what is an expertise conclusion. Of course, neither "science" nor "expertise" is neutral. But expertise, as a decisionaiding tool to giving insights to public decision-making, must capture our vigilance. Michaels (2008) and Oreskes and Conway (2010) have provided significant

\footnotetext{
${ }^{2}$ This distinction seems to be trivial. The reader may argue that the function is obtained by the experience and the scientific/technical knowledge. It is not always the case.
} 
contributions that show, in our point of view, how expertise (and not science) is exploited to defend private interest or self-interest with huge impacts on health, SSE (e.g., cigarette manufacturers strategies or the use of bisphenol A), producing doubt biasing scientific controversy and discrediting the value of public expertise. It may create a discredit on the value, the credibility, the legitimacy and on all others considerations that are expected from public expertise.

Considering these first basic questions and observations, what can our answers be?

Our first answer is that, despite the fact that validity, robustness, legitimacy and coherence of expertise process, methodologies and conclusions, when dealing with SSE issues, are not often patent as well as direct causes of major accidents and disasters, experience feedback still shows that they are in-depth and structural vulnerability factors of public policies in this field. Learning from accidents, means sharing more than a "safety and/or a risk culture" but a "culture of accidents" (Dechy et al. 2010) that helps Experts and Analysts in materializing the outcomes of theirs studies that are rarely accessible for in-lab safety or/and risk prevention theoreticians.

The second answer would be that our concern is of course about a "procedural ethics of expertise" (where rules/norms for the design of processes of expertise are discussed) and not about a "substantive ethics of expertise" (where moral norms of expertise are deducted and explained). Such procedural rules/norms could be focused on the process of expert judgments for policy processes. Indeed, failures in expertise, such as the recent prediction of the Aquila earthquake (2009), can be seen symptomatic of the failure of experts to be concerned by the social and societal implications of their studies and their conclusions. In The imperative of responsibility, Hans Jonas (1985) has proposed an ethic for the future where the future must be the major object of concern. Indeed, given the potential impacts and consequences of expertise conclusions in SSE, we do think that it is an absolute obligation for the expertise community to adopt the consequentialism ${ }^{3}$ doctrine and try to organize the process of expertise by anticipating those consequences, assessing them and grounding their choices on this assessment. Therefore, given these considerations, an ethics of expertise should provide answers about "how to conduct and organize an expertise process? Who should or have to be involved and consulted? When and how should the process be done? What should be done and organized when facing deep uncertainty, dilemmas and opposite, fragmented or contradictory expertise conclusions?".

Our last answer is that questioning the way expertise is performed in SSE has an immediate link with the public policy assessment issues. Expertise is a process that produces outcomes or results and is often summarized or assessed according to them. Another way of paying attention to expertise is to focus on the legitimacy of the expertise process or on the expert's status. In fact, since SSE issues are public and can be collective or of common concern, it is necessary for the public arena to

\footnotetext{
3 We have chosen the "Consequentialism", as a moral doctrine where what count is evaluating an action is its consequences for all the individuals that could be concerned, rather than "deontological" doctrine that evaluates the rightness of an action in terms of its conformity to a norm or a rule, such as the Kantian categorical imperative.
} 
assess and analyze the impact on SSE of the conduct and the governance of the expertise. Testimonies about how expertise is in practice organized and governed in context is a powerful tool to bring to the fore the underlying limits and advantages. Let us notice that the idea of assessing expertise is attractive, but difficult to implement operationally speaking. Indeed, there is a need for both an analytics examination for both methods to implement and the context of expertise and also the need for an ethics that act like a monitoring system or an introspective approach on expertise in SSE.

Decisions, policies and actions in SSE seem in our times to be driven by science where experts are considered as being neutral ${ }^{4}$ by the fact that they are not involved in a decision process and follow scientific codes. They should be scientifically robust in the sense that they frame and they resolve problems according to the best and up to date scientific knowledge.

\subsection{The state-of-the-art: from fragmented contributions to an integrated approach}

The scientific literature in this field, that will be presented hereafter, shows that this question was studied in a fragmented way. In SSE, the problem of the consistency of expertise was studied by the engineering community. Expert judgment is considered as separate from "value judgment" and the main objective is, according to specific case studies, to be able to develop procedures to elicitate expert judgment and to select experts to be a part of a collective expertise process (Goossens et al. 2008). Many protocols and methods were developed to deal with problems such as postaccident investigations or risk prevention. Major contributions were done in the field of nuclear safety (see for example Lannoy and Procaccia 1996) and chemical and petrochemical plants (see for example Cooke and Goossens 2000).

Other authors have focused their attention on the issue of transparency, validation and how to frame more democratic expertise and decision-making processes when dealing with risk analysis and risk management ${ }^{5}$ processes (see for example Renn 1998; Reid 1999; Assmuth and Hilde 2008; Rosqvist 2010). Indeed, since stakeholders' opinions were considered, by a large majority of the engineering community in SSE, as none consistent and too emotional, the main issue was to fight against these ideas. In fact, stakeholders are impacted by the decisions and the conclusions of expertise; they should be consulted and involved in the decision aid and in the decision-making processes. Guidelines and contributions such as Renn (1991a, b), IRGC (2006) and Renn (2008) are central for the scientific and practitioner community in the field of SSE.

Complexities of systems and of decision contexts and situations have also captured the attention. Main contributions were given to deal with these issues and

\footnotetext{
${ }^{4}$ One can think that the time where experts were considered as being neutral is a long time ago. Unfortunately, this is always the "institutional message" that is spread in and out the public expertise institutions.

5 Risk management involves the design and implementation of the actions and remedies required to avoid, reduce, transfer or retain the risks (see "An introduction to the IRGC-Risk GovernanceFramework" published by the IRGC in 2008).
} 
support experts on choosing the right models (Gertman et al. 1996; Horlick-Jones 1998; Lagergren 1998; Amendola 2001; Fairbrother et al. 2007).

The characteristics of the accidents investigation domain in SSE has enlighten the difficulties faced when dealing with tricky and strategic decision situations such as attributing responsibility (or even blame) for juridical needs. Analysts/Experts are often under political and administrative constraints in their process of determining causal links. In these circumstances, it is often advocated that they should be independent from justice, authorities and businesses but competent enough which sets a stable dilemma (ESReDA 2009, Dechy et al. 2012a, b; Dien et al. 2012). In the same subject, Llory (2000) has explored some engineering ethical questioning when performing a risk analysis in socio-technical systems and has given some insights based on experience of major accidents.

More largely then in the field of SSE, many authors have focused their attention on the issue of how to make choices and decisions and how to reduce biases.

Strategies to prevent perception biases were suggested; see for example, the four strategies proposed by Fischhoff (in Kahneman et al. 1982) ${ }^{6}$ or the works done by Stanovich and West (2000) ${ }^{7}$. Epstein (1994) and Slovic et al. (2002) have suggested a way to move from an intuitive mode of reasoning to an analytical one where Kahneman and Lovallo (1993) have suggested strategy that consists in taking into account the point of view of an outside actor. Other strategies were suggested that encourage the actors to take a contrary view to allow them to reconsider their choice conditions or to understand the process of cognition and what follows in terms of biases, enabling them to reduce in consequence the biases (see Slovic and Fischoff 1977 and Fischhoff in Kahneman et al. 1982).

In Merad (2010), we proposed a methodology to support the conduct of expertise in risk analysis and risk management based primarily (mainly?) on the idea that the fact of putting into perspective the expertise driving conditions enables to reduce biases. Even if the expertise can be regarded as public decision aiding (Fig. 1), the expertise process itself needs a support in the sense of a decision aiding.

We have then proposed a methodological approach to fill out the conditions of a participative and deliberative model of expertise (see Merad et al. 2011) (see Sect. 1, A). We have considered that the expert performs in his work of expertise, in a conscious manner or not, a set of choices, based on his level of knowledge, his level of experience, his culture, the context of its intervention, etc. The expert is therefore, in the process of analysis, individually or collectively, in a manner akin to a decision-maker (in the sense that he has the power to define the framework and the terms of the expertise process and is responsible for the technical credibility of its findings) (Fig. 2).

\footnotetext{
${ }^{6}$ (1) implements the alerts on the possibility of bias, (2) describes the direction and the sense of the bias, (3) provides feedback, and (4) implements a training program with experience feedback, coaching and other interventions in order to improve the judgment.

7 The authors suggested distinguishing the system 1, which refers to an intuitive system that is fast, automatic, effortless, implicit and emotional, from the system 2 which is more reasoned, slower, more aware, more explicit in its application efforts and is regarded as logic. The great difficulty is to switch the actors of the system 1 to system 2. Different strategies are then possible.
} 

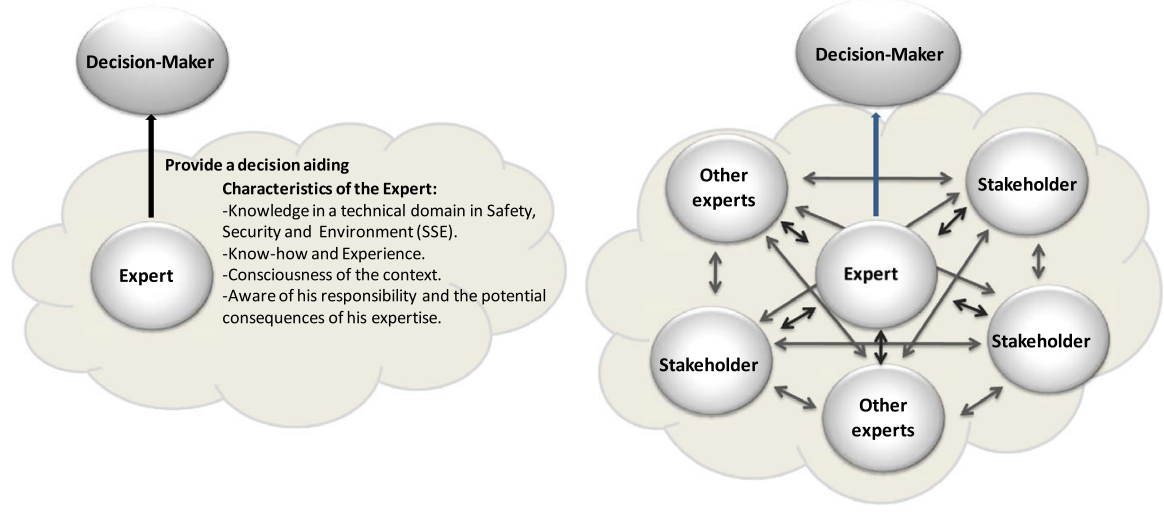

Fig. 1 Expertise in SSE as a decision-aiding process for decision-maker (Left situation of expertise in SSE with an Expert and a decision-maker, Right more complex situation in expertise)

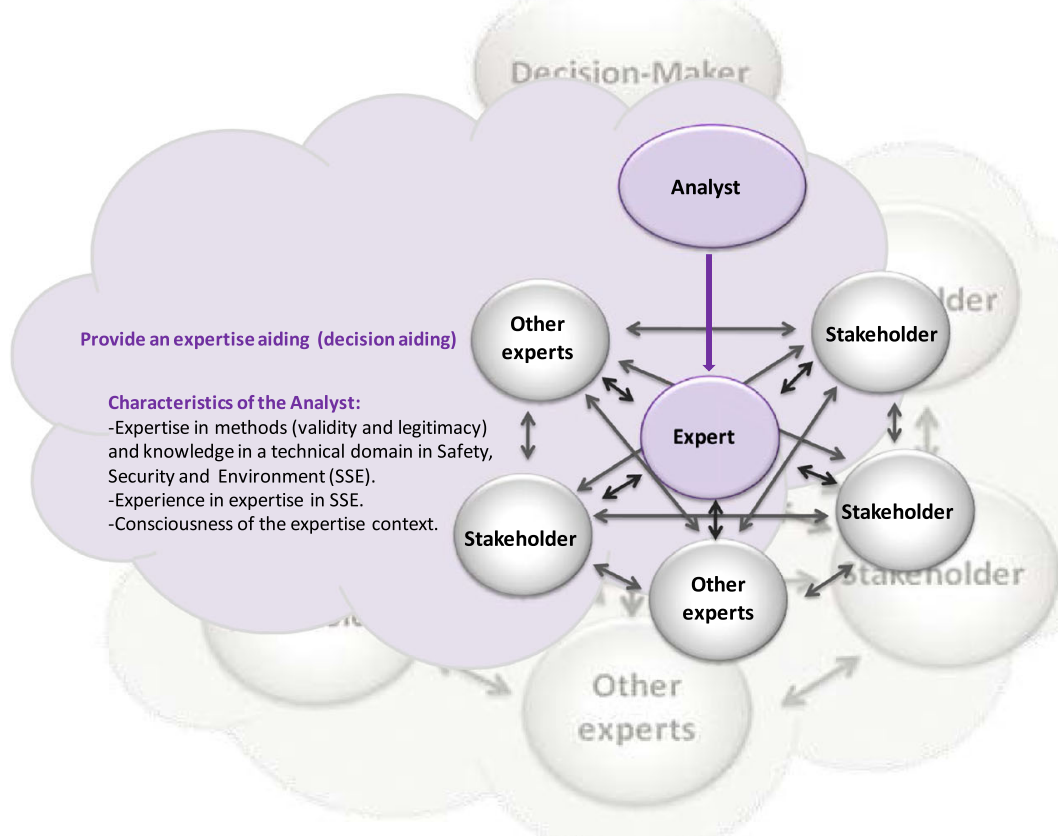

Fig. 2 Expertise in SSE: a need for a decision-aiding process for experts

In this paper, we suggest that there is a need for an integrated framework of expertise analytics and expertise ethics (see Fig. 3) that should be based on: (1) experience feedback about practical experiences of the practice and governance of 
expertise in SSE to explicit the lacks, failures and the practices that were identified and developed in context, (2) explicit observations and recommendations. This last point must be distinguished from procedures. In fact, we do not want to develop norms about expertise such as the NF X 50-developed by AFNOR (2003). Our purpose is to provide, for both public expertise practitioners and stakeholders a framework to assess and appraise the conduct and the governance of a public expertise in SSE and develop a critical lecture of aptitudes and attitudes before, during and after an expertise process. Indeed, we would like to provide an integrated framework that is endogenous to the public risk decision world and not only exogenous, like norms used to be for example.

Sharing experiences on failures and successes of expertise in SSE is fundamental but not easy to get. This can have many explanations depending on the culture and the regulatory constraints within a country. In fact, expertise is a part of the regulatory system in SSE; and SSEs are common and public concerns. Therefore, pointing topics such as potential lacks or biases in the governance or the conduct of expertise can be considered as critics to the public decision-makers. Let us consider, for example, the disparity of practices when coming to the sharing of experiences about accidents investigation. Some countries are more transparent than others and are more prone to provide easily documentation to the general public (Llory and et Montmayeul 2010; Merad 2010).

When being involved in and/or conducting a public expertise in SSE, experts and analysts are not facing a theoretical simplified in vitro situation but are imbedded in vivo in complex situations with a multiplicity of constraints and components that cannot be easily isolated. They are discussed in Sect. 2.

\section{Public expertise: organization, problems and conclusions}

When dealing with high-risk industries or natural hazards with potential catastrophic outcomes, they often become more regulated after major accidents and disasters (see for example, post AZF Toulouse disaster in September 2001 in Dechy et al. (2004), and the promulgation of a risk law in France in July 2003 in Merad and Dechy (2010)). SSE are public common goods. Depending on countries, the State organizes, according to his administrative and local culture, a way to protect the citizens and their goods from short, middle and long-term potential harms. Public expertise is then institutionalized and organized to explicit and to assess the risks induced by a technology (e.g., implementation of a chemical or petrochemical plant) or induced by natural hazards (e.g., flooding, earthquake). Let us share an example on how the public expertise is schematically organized in France.

In France, public expertise in SSE is divided into different agencies. Each agency has a specific field of competence (e.g., nuclear, health, chemical and petrochemical, etc.,) and is under the supervision of one, two or several ministries. These agencies are funded by the public sector, can have different legal statuses allowing them to carry different activities (such as public expertise, research and commercial activities) and can be balanced by private and public funds. Public expertise 


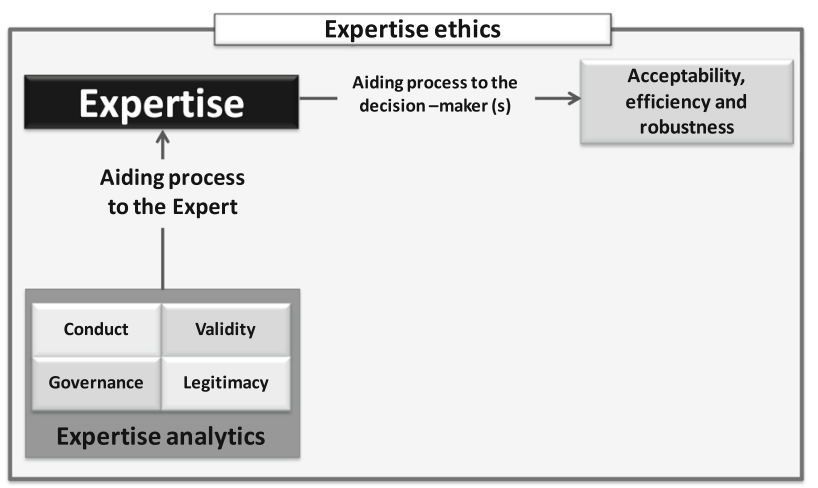

Fig. 3 Analytics and ethics of expertise: an integrated framework

agencies support ministries in framing laws and national risk prevention guidelines, anticipating future problems and developing solutions by conducting research activities. Depending on their status, public expertise agencies can have the possibility of self-referring (auto-mandate) when a SSE problem occurs in the public arena (Fig. 4). But, in the large majority of cases, these agencies are mandated by the State, or by the ministry that supervise the agency, or by the inspection (that is in charge of auditing and controlling the correct application of the regulation in SSE). If a private stakeholder (ex. Industrial operator) asks for an expertise to a public agency, this is done in the context of a business contract.

In UK or in USA, public expertises in SSE are not always similar to those made in the French context. Research is sometimes separated from the main expertise done for the regulation activities. Inspection and expertise can be grouped in the same organization (see for example in the UK, the HSL and the HSE, the EPA and the NRC in USA).

Public expertise institutes or agencies can perform individual or collective expertise to give a decision-aiding support to the public authorities. Let us notice that agencies differ from institution by the fact that they have objectives in terms of risk reduction and prevention, and institutions must contribute to risk prevention. The expertise is called individual when a member of the agency is requested for his/ her scientific or technical knowledge to inform solely the public decision-making. The knowledge mobilized for the expertise is in majority from one or multiple SSE scientific and regulatory domains and/or as well as for the methodological knowledge on the way of organizing risk assessment, risk management and/or risk communication processes. In a majority of situations, the expertise is untitled as collective. That means that multiple domains and/or methodological experts are mobilized to enlighten the public decision-making in SSE.

As a part of the SSE public decision process, the expertise process is a decisionaiding process that is neither linear nor static. This last process is under the influence of public decision-makers, scientists, industrial operators, NGO, mayors, citizens, other expertise organizations and on the other side, to the stakeholders. 


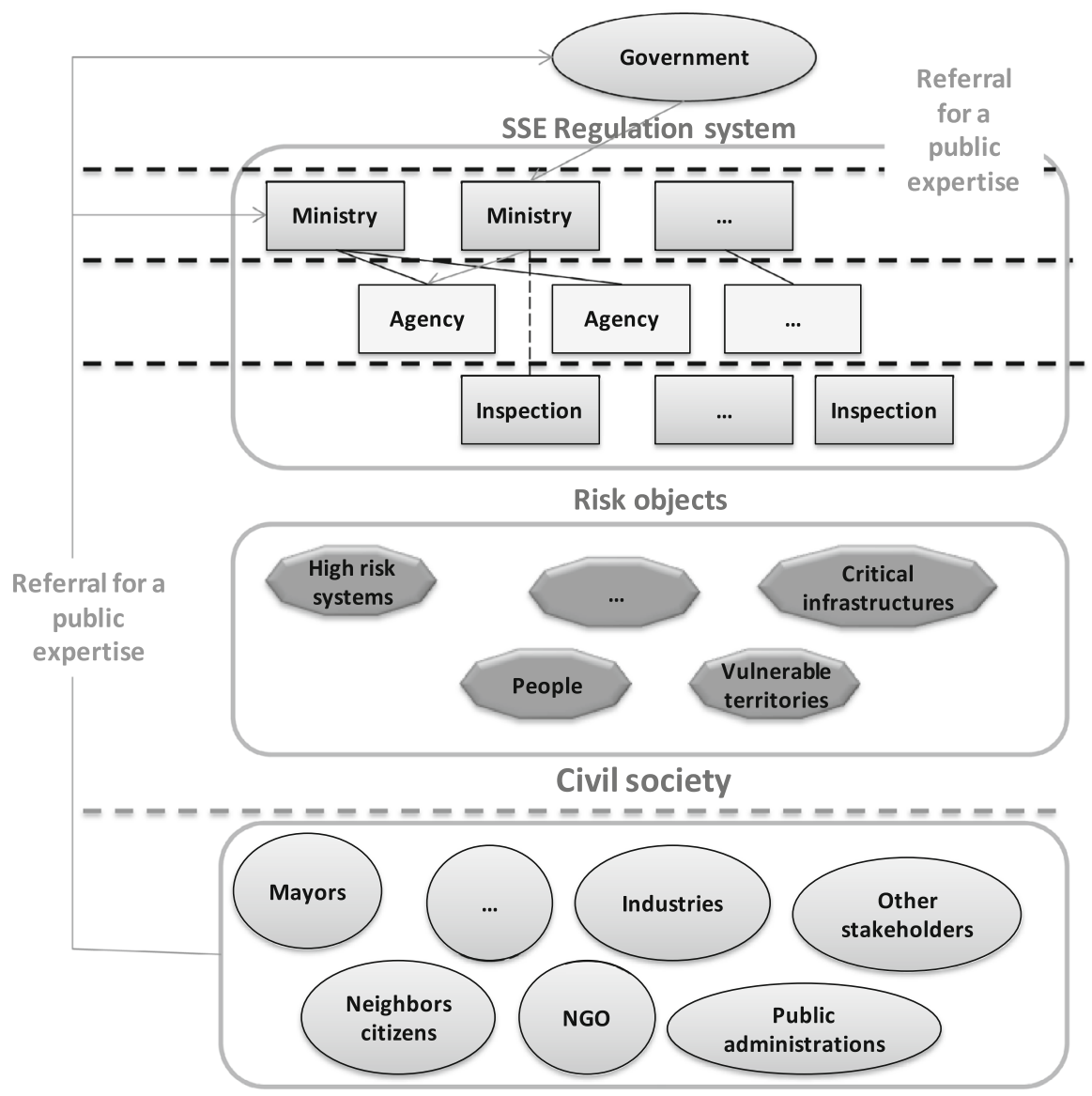

Fig. 4 SSE public expertise in France

\subsection{Three models of expertise and situations of interaction between actors}

In Merad et al. (2011), we suggested four levels of stakeholder participation (information, consultation, association and deliberation) according to the impact level of stakeholder participation on the final decision-making and to the level of equality between the decision-maker (DM) and other stakeholders. This allows distinguishing three models of expertise that can give an interesting insight on the way public expertise is organized in different countries.

Model I is based on an information model of public participation where there is a strict separation between risk assessment, risk management and risk communication. This model was and is still dominant in France and in several other countries. In this model, we face classical interaction situations in SSE that consists in biactors situation: a decision-maker (and/or his contractor) that comes in with a formulated issue or problem and an expertise agency that is asked for bringing a practical technical solution. 
Model II is based on a consultative framework of public participation where specification on the way risk is assessed is given by explicating the underlying arguments and the collectives' rules of risk framing. In this second model, expertise should provide a possibility to be audited and should be transparent. Procedures must then be traceable and experts must be independent. Since the ratification of the Aarhus on the Access to Information, Public Participation in decision-making and Access to Justice in Environmental Matters that was adopted on 25th June 1998, public expertise agencies aim at following this model of expertise. In France, this model was retranscribed in a more proceduralized expertise: institutionalization of quality assurance system, set up of deontology committees and promulgation of deontology procedures and booklets, involvement of NGO on the board of director and scientific board meetings and promulgation of charter of "opening of expertise to civil society" 8 . This tentative of transformation of expertise towards more openness to civil society was strongly influenced by the Canadian model such as Environment Canada. However, the proceduralization of expertise is rather due to the French administrative culture and mostly influenced by the application of the ISO 9001. This model presents different interaction situations in SSE, including the situation presented in model I and the following situations:

- $\mathrm{DM}$ is in interaction with the Expert (Analyst). Other actors/stakeholders are concerned and impacted by the SSE issue. The Expert, or the DM, is in charge of considering their opinions, preferences and their expectations. The Expert can be expert in a specific domain in SSE or a methodological expert (e.g., facilitator). The expert is asked to bring a practical solution, or/and to reduce the level of uncertainty and/or ambiguity on a specific situation

- DM and different actors present an issue to the Expert (methodology, domains). Expertise agency is asked to frame SSE issues and sometimes to switch from giving recommendations to making the decision.

In the model III, expertise is based on a participative and deliberative framework of public participation that includes the model II of expertise. Questions such as legitimacy and validity of procedures and experts knowledge are raised with respect to their limits and to the way they were produced. Until now, this approach has not faced a big success. Maybe due to the fact that it is difficult to find a way that makes it technically and organizationally operational, effective and efficient. In this paper, we will give a contribution to this model.

\subsection{What is the problem?}

Studying how public problems are framed in SSE is not a trivial task. Some problems are explicitly raised by a DM after an event [e.g., accident investigation after the explosion of an ammonium nitrate chemical plant in Toulouse (France) in 2001] or due to new regulatory constraints (e.g., land-use planning around Seveso

\footnotetext{
${ }^{8}$ See for example the charter signed by the expertise institutions such as IRSN, INERIS, CEMAGREF (IRSTEA), IFSTTAR and ANSES: http://www.irsn.fr/FR/Actualites_presse/Communiques_et_dossiers_ de_presse/Pages/20110909_Charte-ouverture-societe_expertise_inter-instituts.aspx.
} 
Plants due to the application of Seveso II directive and the French national law on risks prevention 30th July 2003), while some others are the result of a social dynamism following controversies, conflicts or whistleblower alerts (e.g., asbestos, nanotechnologies or bisphenol A). These problems are risk problems that can be simple, complex, uncertain or ambiguous (IRGC 2006; Merad 2010). They can have an impact on a local, regional, national or extra-national scale; they can involve and/ or impact a few actors or a large set of actors. In fact, expertise problems in SSE are delimited in an explicit or implicit contract between the expert/analyst and the DM (Fig. 5). In this contract, the DM gives his appraisal of the perceived risk problem. The Analyst must give his recommendations according to the perceived risk problem fixed in the contract.

The main starting difficulty for the expert/analyst is to be able to frame a coperception of the risk problem. This is not always the case (Table 1). When perception is identical (Situation C, Table 1), the contract is well framed which means that the resources are adjusted according to the constraints. When the gap in perception, denoted $\Delta$ Risk perc, is significant, the constraints and stakes on the contract can be over (situation A, Table 1) or under estimated (situation B, Table 1).

In situations $\mathrm{A}$ or $\mathrm{B}$, experts will have to take more time stating and framing the contractual expertise context with the DM and the underlying impact on the conduct of the expertise process. Let us remark that even if the contract between the DM and the expert is a preliminary condition to start the expertise, the interaction throughout the expertise process will make the problem evolve with time and even change in nature. Indeed, the expertise process is a diachronic process that should be traceable.

Adapted framing, sound, accepted and robust conclusions of expertise will then depend on two major conditions: validity and legitimacy. These two conditions will be explicated hereafter.

\subsection{Conclusions and recommendations: What can be the outcomes of the expertise process?}

Public SSE expertise is a decision-aiding process. In that sense, the outcome of the expertise process is neither a decision-making action (e.g., expropriation when houses are in the red hazard zone at the vicinity of a hazardous plant and submitted to explosion phenomena) nor a scientific evidence. Outcomes are recommendations, conclusions that can be prescriptive (e.g., according to these observations, the set of measures that should be considered to improve safety) or explicative (e.g., accidents database provide heterogeneous information, the method used to assess the probability of accident scenarios is different from a plant to another) that could have impacts on different territorial, social, economical or environmental scales, which could then concern variable number of actors. As risk problems, expertise conclusions can take different aspects:

- Information: This aspect can be, for example, a level of probability of the occurrence of a dreaded event such as explosion of an industrial plant, for example, or the impact distance with lethal effects. It can also be an observation 


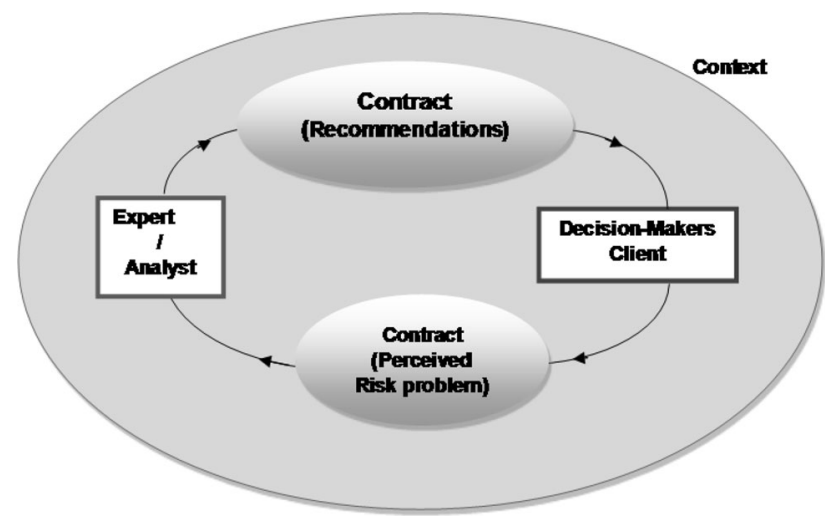

Fig. 5 How is the risk problem framed? A contract between the expert and the decision-maker (DM)

Table 1 Gap of perception $\left(\Delta_{\text {Risk perc }}\right)$, between the DMs and the Experts of risk problem

\begin{tabular}{lllll}
\hline & \multicolumn{2}{l}{ Perceived risk problem by the expert } & \\
\cline { 2 - 4 } & Simple & Complex & Uncertainty & Ambiguity \\
\hline $\begin{array}{l}\text { Perceived risk problem by the DM } \\
\text { Simple }\end{array}$ & Situation C & & Situation B \\
$\begin{array}{l}\text { Complex } \\
\text { Uncertainty }\end{array}$ & Situation A & & \\
Ambiguity & & Situation C & Situation C & Situation C \\
\hline
\end{tabular}

such as, for example, conformity or not to ongoing standards in safety within a chemical plant (conformity or not).

- Diagnosis or/and analysis: Post-accident investigations are an example (see examples in Dechy et al. 2011, 2012b).

- Approach, methodology, method, process, procedure or tool: They can be for example the development of a national guideline for land-use planning around Seveso High Threshold plants (see for example Merad et al. 2008).

These expertise conclusions depend on the nature of the risk problem (see Table 1). The less the risk problem is perceived as simple by the DMs, the more the public expertise process is piecemeal framed. Moreover, the final conclusion is based on fragmented sub-expertise conclusions that are seldom connected.

\section{Towards a generic integrated framework to an analytics and an ethics of expertise}

Being involved in SSE public expertise and sharing experiences on SSE expertise have helped us to address several kinds of issues. That is why we suggest a generic integrated framework (Fig. 6) aiding at: (1) expliciting the way expertise is 
conducted and governed to be able to identify a set of biases and constraints, and (2) suggesting some prescriptions to frame valid and legitimate expertise conclusions. We mainly framed our approach by being strongly influenced by the grounded theory (Martin et al. 1986; Allan 2003; Kelle 2005; Thomas and James 2006) and by the works done by David (2001) that have contributed to provide a theoretical basis to the conception-task.

Figure 6 provides an overview of our proposal of necessary aspects to consider when dealing with expertise issues.

To understand the way expertise is conducted and governed, it is useful to explicit three main aspects that define expertise:

- The expert characteristics: Who is mandated (a group, an institution...)? What are their scientific and technical cultures? How is expertise organized and managed?...

- The context and its characteristics: Who are the actors involved including the DM? What are the sets of stakes and constraints (scientific, technical, economical, regulatory, media...)?...

- The methods: What kind of approaches, models and tools are used? What kind of disciplines are mobilized? What are the limits of their approaches?...

The triptych defined by the interactions between [Expert (Analyst)-the contextthe methods] fixes the conditions of validity and legitimacy of expertise. When dealing with SSE, analysts are not often free to reframe their mandate or their contractual conditions even if it is necessary. In fact, strong contextual and internal constraints such as regulations (e.g., laws) and procedures (e.g., good practices) should be respected. Moreover, the legitimacy and the validity of the expertise process and of the conclusions depend not only on the precision of the technical data and knowledge, but are also directly or indirectly influenced by a multitude of other

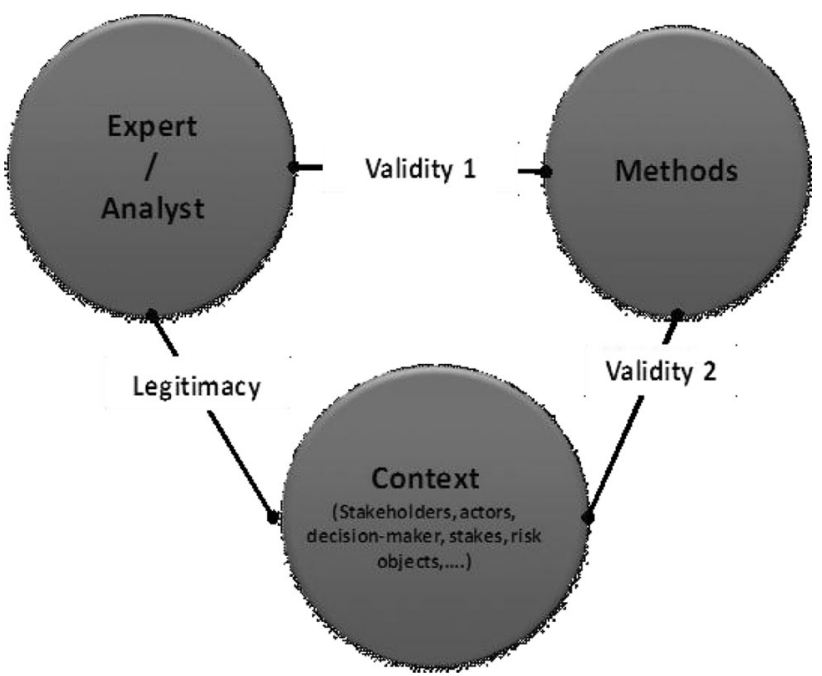

Fig. 6 Integrated framework for expertise analytics in the field of SSE 
dimensions such as financial, social, legal aspects, etc. These interacting dimensions are sometimes difficult to predict, to explicit and their impacts remain hard to qualify.

Thus, experts have to explicit, before, during and after a study, what were their choices, their assumptions and preferences knowing the set of limits and constraints they were subject to, what are the uncertainties about their data and what is the robustness of their conclusions. Let us say that expertise is submitted to a constraint field (see Fig. 7) with a large set of constraints that must be considered.

In what follow, we have made a distinction between the conduct of expertise and the governance of expertise (see Fig. 8).

\subsection{Some insights about validity and legitimacy conditions}

As we previously stated for identifying and fixing expertise problems, choosing an expertise method and framing conclusions are critical issues. Indeed, there is now a need to pay attention to the way problems are framed and to the way methods are chosen to frame recommendations.

\subsubsection{About validation}

Insuring the liability, the consistency, the robustness, synthesized as the validity, of expertise means that there is a need to explicit the context of the conduct of expertise, the biases on the framing of expertise and those induced by methods that are chosen and the limits and robustness of expertise conclusions. The decision aid literature has contributed to give interesting insights about the validation process that should be used by the Analyst (see Landry et al. 1983). Four aspects are suggested:

Fig. 7 SSE expertise under a constraint field

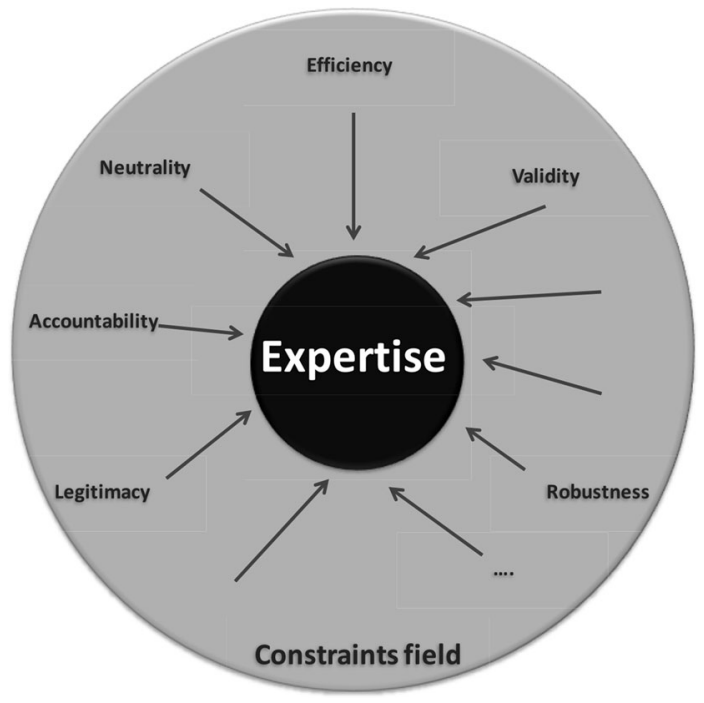


Fig. 8 Conduct and governance of public expertise process in SSE under validity and legitimacy oversight

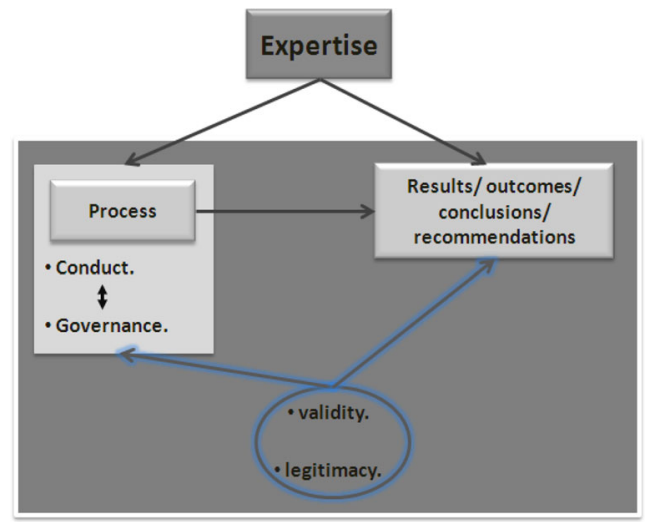

- the conceptual validation,

- the validation of the logical consistency of the model,

- the experimental validation by using data coming from real life situations, and

- the operational validation that consists in following the daily life of the model.

When dealing with SSE and risk problems, what can be transferred and adapted about validation? In the following, we have distinguished two categories of validation:

- Validation of category 1 (Validation 1) that includes the conceptual and the logical validations (see Fig. 6), and

- The validation of category 2 (Validation 2) that includes the experimental and the operational validations (see Fig. 6).

Expertise conclusions must effectively contribute to reduce risks on health, on environment and on goods. The DMs and other impacted and/or involved stakeholders should understand both the process of expertise and the conclusions in terms of added value and limits. This operational validation can be obtained only ex post. On the other hand (However?), organizational measures can be taken ex ante and during the process of expertise to ensure achieving this kind of validation. Such measures can be undertaken to better understand the context of expertise and to learn from experience feedbacks from practices (successes and/or failures) in SSE expertise processes. This experience feedback can be of different kinds: sociological and quasi-ethnological descriptions, such as for example, the description of the Challenger launch decision by NASA as described in Vaughan (1996), or some more compact and resumed descriptions such as, for example, the organizational investigations of accidents in Llory and et Montmayeul (2010). This last point can also contribute to the experimental validation.

But, it is never easy to benefit from the sharing of failures in SSE expertise processes. First, because there is a reluctance to point out these aspects and then because there can be some significant juridical/legal, political and societal impacts. More discussions about these aspects can be found on Dechy et al. (2011, 2012b), Llory and et Montmayeul (2010) and Cicolella and Benoit-Browaey (2005). Let us, 
for example, point out the AZF Toulouse expertise (Jacob and Seillan 2012) where, more than 12 years after the major accident, it is still difficult to benefit from transparent and public documents on how the expertise was conducted and what were the different scenarios of accidents that were identified and not considered; or let us discuss about the way risks induced by asbestos were recognized in France, and how whistleblowers working in expertise public institutions were reduced to silence (Chateauraynaud and Torny 1999).

Norms, rules, regulation, good practices, etc., are numerous in SSE. They depend on the nature of risk, the sector of activity (e.g., chemical and petrochemical, nuclear,...), the national and/or organizational culture. Indeed, conceptual validation can be subdivided into two categories: validation of fixed concepts and validation of consensual concepts (soft laws). Let us illustrate our last point by a French example on the way land-use planning was defined on territories above mines. A risk zoning was done by an expertise agency on 1999 based on an ongoing definition and conceptualization of the risk of mining subsidence defined as a function of the probability of occurrence of a subsidence and of an expert judgment (meaning qualitative assessment) on the sensitivity of the stability of the configurations of mining exploitation. Three years after, the consensus on risk definition had changed. Risk was then defined as a combination between the hazard (collapse and subsidence) and the vulnerability at stake. More precisions about the example can be found in Merad (2010). Risk is in this example a consensual concept that changes during time, which makes the conceptual validation difficult to obtain for the whole expertise process.

Of course, some references of this conceptual validation can be obtained by looking at some aspects of the expertise process that are based on some mathematical, chemical or physical stability concepts. Indeed, conceptual validation is hardly applicable to public SSE expertise process. Last but not least, let us finish with the validation of the logical consistency of the model. Experience feedback shows once again that it is complicated to find an expert that excel in "methods based expertise" or in "system based expertise", but it is very hard to find an expert who excels in knowing both systems and methods. Let us give for example, the systematic errors that are done when assessing the probability of occurrence of accident scenarios during the framing of safety studies for chemical plants. Indeed, the problem of logical validation could be tackled by framing adapted training programs that help framing a culture of methods and systems at the same time.

\subsubsection{About legitimacy}

Now, how about the legitimacy of public expertise in SSE? This aspect is considered in the way an analyst will:

- Try to consider the contextual and the organizational condition of the problem's emergence as stated by his client. Tsoukiàs (2007) suggested that the way the problem is stated can be considered as a pretext to start the decision aid process.

- Take care in building a relationship between the client and himself, similarly to the relation between a patient and his doctor, by the fact that the relationship 
between the two is legitimate by the time that is invested by the doctor in an active listening.

- Take care of the impact of the recommendations after all.

Considering contextual and organizational aspects seems to be of equivalent importance in both validity conditions and legitimacy conditions. How about risk problems? Let us point out that in public expertise in SSE, the risk problem is, in the majority of cases subject to a contractual agreement. If this one is verbal, it is possible to consider the risk problem stated by the DMs (or his representative) as an attention catcher or introduction to the expertise process. This is rarely the case. The contractual agreement is more often written, the expert is directly or indirectly more or less subordinate to the DM (e.g., public authority) and, even if it is possible to discuss the limit of the problem in a research project or in some ad hoc committees and commissions, reframing the limits of the risk problem is better to be one of the given conclusions to the DM, in addition to giving an answer to his stated risk problem, then as a starting point of the expertise process. Let us say that we do think that considering contextual and organizational conditions and reframing the risk problem will be useful for the expert/analyst considering deontological and practical expertise concerns.

Investing time in building a relationship between the expert and the DM is of course fundamental. Mainly because it will help to reach a convergence in the perception of the risk problem (see Table 1), and it will also help in establishing a common vision of the stakes, the means and the constraints that will occur during the expertise process. But this relationship must not be limited to these two parties. Other stakeholders should and must be included in this time framing. Firstly, because that will help to consider other aspects of the problem that were not identified and pointed out initially, deliberately or not, by the DM. Secondly, due to the fact that even if the client is the DM himself or his representative, the SSE public expertise's real client is neither tangible nor immediately visible, but is the main thing in public services "working to prevent risks for citizens/peoples and ensure health, safety and security with respect to environment". Therefore, other actors and stakeholders should be, or let us say must be, involved and consulted. Let us finally point out a limitation induced by the interaction between an Expert and a DM (like Analyst and his patient) that consists in an imposed or a co-framed (simultaneous) blindness on the framing of problems and on the consciousness of the potential impacts of expertise conclusions. In SSE public expertise, many examples can be done and have an influence on the limitation of the risk problem framing, the organizing of the expertise process and framing conclusions. The story of the Minamata disease ${ }^{9}$, which is the most severe methyl mercury poisoning and one of the most severe health disasters of the history of chemical industry that occurred in the 1950s and 1960s, caused by man-made environmental pollution, is an illustrative example. Many other lessons can be of course learned from the Minamata disaster.

\footnotetext{
9 To learn more about this disaster, the reader can refers to Mishima (1992) and Sakamoto et al. (2010).
} 
Taking care of the impacts of expertise conclusions can be obtained by considering the context of expertise and decision-making in public SSE risk problem. Unfortunately, let us point out that some ongoing norms (see NF X 50-110 of AFNOR) in expertise do not seem to be prone to this idea. Why? Because it is a common argument to presuppose that strict separation between expertise and decision and their impacts will increase the neutrality and then the legitimacy of expertise conclusions.

After all and in other words, what can we suggest to increase the quality and the reliability of both the conduct and the governance of public SSE expertise? Let us resume our discussion hereafter (see Table 2) on how to incorporate the two major principles which are validity and legitimacy.

In what follows, we will give some insights on a crucial task that was pointed out in this section: how to explicit the context of expertise to improve the quality of risk problem framing and conclusions framing, but at the same time to ensure the quality of the expertise process as a whole.

\subsection{Understanding context, facilitating expertise process}

The decision aid literature has given a large contribution on this subject (see for example, Vincke 1986; Al-Shemmeri et al. 1997; Georgopoulou et al. 1997; Guitouni and Martel 1998; O’Keefe 1989; Roy 1985; Tsoukiàs 2008). Some references focus on the way of making the decision process more coherent by looking at the methodological and deontological aspects of decision aid (David 2001; Tsoukiàs 2008). In SSE, however, the role and the difficulties faced by the expert/analyst in practice are not well detailed. A different perspective was considered by looking at the research done by Wisner $(1982,1995)$ in ergonomics. Ergonomic is focused on the way the work is done in a given situation. Indeed, by looking at Wisner's works, we have to look at the "real working situations" of the Analyst/expert (that means to the context and the methods that he uses) to be able to understand the work done by him. In fact, in real life situations, expertise problems do not pre-exist with the possibility of using methods and framing conclusions. There is a multitude of problems with a multitude of possible solutions and conclusions. The expert should build compromises between contradictory objectives and should develop what Wisner calls an "understanding and intelligibility of practices"10. Extrapolating Wisner's proposal to expertise contexts means that making the context of expertise explicit is done by identifying regularities and laws that can organize the appearing untidy of the analyzed' situation or problem. According to these proposals, we have made the hypothesis as practitioners of expertise in SSE that the difficulties faced by an Expert/Analyst can be due to a misconception of the constraints on the subject of expertise process or "decision aid" process. In fact, each SSE problem is a project under constraints fixed by the Organization and its direct environment (context). These constraints are aspects that help to frame the SSE problem.

\footnotetext{
$\overline{10}$ "Une intelligence de la pratique et avoir recours au concept de la mètis".
} 


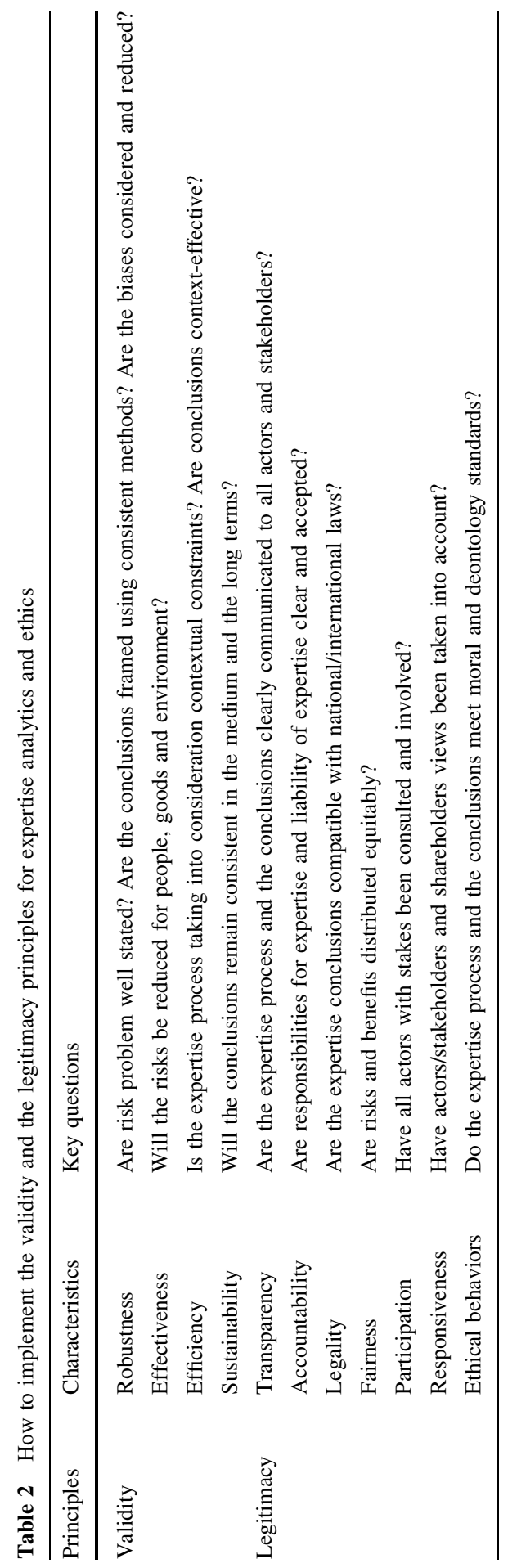


At this stage, if we admit that "expertise is a decision-aiding process to the DMs" and that "expertise is also a decision process that needs a decision-aiding process to expert(s)", it is then useful to consider some contribution to analysts in designing its process.

Tsoukiàs (2007) has suggested a multiple-step process. The first step of this decision aid process consists in the framing of the problem situation $P$, where $P=\langle A, O, S\rangle$. The set $A$ is the set of actors of the decision process, $O$ is the set of stakes that actors want to introduce in the decision process and $S$ the set of resources that the actors introduce in the decision process. $P$ is framed during the interaction process between the DM and the analyst. We do think that, when dealing with SSE expertise process, $P$ should not only be the result of an interaction between the DM and the Expert, mainly because of problems of neutrality/partiality (even if DM can be the public authority), responsibility and more largely because of problems of legitimacy, but mostly be the result of a larger interaction between the Expert, the DM and the other stakeholders.

Then, we propose an adaptation with $P=\left\langle C_{\text {contExp }}, \Delta_{\text {Riskperc }}, B\right\rangle$ where $C_{\text {cont Exp }}$ is the set of contextual characteristics identified by the Expert; $\Delta_{\text {Risk perc }}$ is the gap in risk problem perception between the expert DM and the Expert (see Table 1); and $B$ is the set of cognitive, collective or organizational biases and factors that influence risk perception.

\subsubsection{The five contextual characteristics $\left(C_{\text {cont Exp }}\right)$ that influence the conduct of expertise}

In what follows, we have identified five contextual characteristics that influence the conduct of expertise (Fig. 9).

1 The level of the decision-making problem. Expertise is a process in a SSE public decision process. Expert has to identify the level of decision his Client and the DM are involved in and their constraints. We have identified three levels of public decision-making concerns: strategic, tactical and operational levels. These levels are distinguished according to the available level of information and to the impacts of the actions taken following the recommendations.

In cases where risk problems extend over long periods, the decisions reached are often found to be cross-functional in nature compared with the three levels of public decision-making.

2 Information and knowledge. The way both the decision-making problems and the expertise problems (risk problem) are formulated is the result of a cognitive

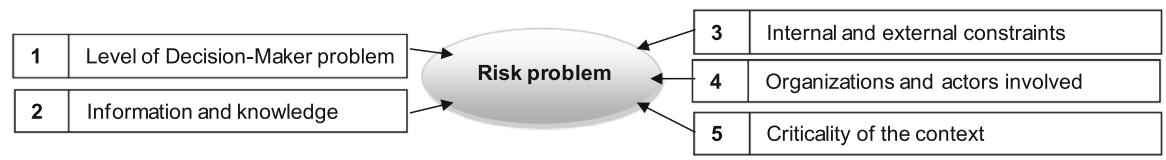

Fig. 9 Five contextual characteristics that influence the conduct of expertise $\left(C_{\text {cont Exp }}\right)$ 
construction where the actors and shareholders involved in the process attempt to draw on their own experience and their own knowledge. Indeed, both the expert/analyst and the other actors involved, including the DM, are in a continual learning process; therefore, we admit that the risk problem is context related.

3 Internal and external constraints: space, time and strategic aspects. Considering the assumption that an expertise is managed like a project within an organization, we observe that it is subject both internally and externally to constraints of time (e.g., deadlines) and resources (e.g., the number of people involved in the study, computer, technical, budget resources, etc.,). It is then essential to explicit these conditions that can vary across time. What we point out here are the well-known constraints of project management. Besides, an expert should pay much attention to consistency between the risk problem and cultural practices within the Organization in terms of "project management" that can be a sine qua non condition for the acceptance of the Expert's approach.

By the term culture we mean "all of the beliefs, behaviors and assumptions shared by a group and resulting from past experience”. For Mintzberg (1989), every organization has its own way of organizing the management of a project and he states that "[culture is] a rich, highly developed and deeply engrained system of values and beliefs that distinguishes a given organization from all others". The word culture is applicable for an organization (e.g., Expertise agency), which means that people may share common objectives (i.e., context of debate). However, if we consider the organization as a part of society, the problem could become more complex, because people may have opposite concerns and are likely to disagree with objectives (i.e., context of negotiation). The problem remains tractable as long as people are open to negotiate.

As pointed out above, organizations such as expertise organizations do not have the resources to collect all information to reach an optimal expertise conclusion. But the problem is even deeper. Even if this information was available, experts and decision-makers would not be able to process the whole amount of information. This is what H. Simon called "bounded" rationality, which constrains the decisionmakers to envisage the finality of decision-aiding differently. It also constrains the analyst to start from a naïve and "positivist" vision of the "best solution".

4 Organizations and the actors involved in or impacted by the expertise process and/or the SSE public decision process. An expertise is conducted in interaction with those who are called actors (Roy 1985). Various categories of stakeholders and actors are involved and impacted by the risk problem and the expertise conclusions. These actors may be the one who asked for the expertise, the person or the organization in charge of the expertise, the experts individually and also various corporate entities or private actors directly or indirectly involved. It is interesting to note that the concept of an actor is neither absolute nor neutral; this presupposes the presence of an observer (expert/analyst) who, based on their problem framing, produces a representation of the explicit or implicit distributions of roles to all of the actors. 
Actors can be grouped in five categories depending on whether or not they hold any power or stake over the final decision (decision-makers), whether their intervention directly influences the expertise (stakeholders), whether they are subject to or intervene indirectly in the expertise (affected by their conclusions), whether they intervene indirectly but are not affected by the consequences of the decision made (ghost or latent actors), or whether they are intermediaries (expertise' requesting party or customers, expert or analyst, advisor, negotiator, referee, informant). Whether for affected parties or stakeholders, it is important in the expertise process to be aware of and explicitly define the roles, responsibilities and interactions between the actors. Based on this observation, the stakes of both the finality and the conclusions of expertise can become clearer to the Analyst/expert.

5 The criticality of the context. Based on our experience feedback as practitioners, expertise process will be influenced by the degree of criticality in the environmental context:

Pre-crisis or pre-accidental. The expertise during this phase must be performed from a preventive point of view. This means identifying and detailing the responsibilities (meaning who will do what) of the various actors involved and identifying and analyzing context related factors.

Crisis or accidental situation. This phase is characterized by a highly dynamic context where effective measures, which need to be taken urgently come together with precautionary principles. We feel that here, the expertise aims more to act on the risk than to understand it. To this end, the methods used should take into account the significant influence of political stakeholders and their impact on modeling the situation. It is also necessary to organize the communication process around the expertise' conclusions towards an audience that is not necessarily specialized to meet a social demand and protect the various stakeholders involved in risk management.

Post-crisis or post-accidental. This phase must necessarily take into account the need to implement measures for repairing and compensating for damage as well as the need to understand and draw conclusions from the events. To this end, expertise performed during this phase aims more to identify, estimate and evaluate risk.

The five aspects above can give an enlightenment to an expert/analyst and can help him to have a reflexive approach on the relationship (the contract) that he has with the Client (who can be the decision-maker) and thus before proposing a method or a tool, to give recommendations (Fig. 7).

\subsubsection{Some insights about the set of cognitive, collective or organizational biases and factors that influence risk perception (set B)}

The set B has captured the attention of many in decision science but also in safety and risks sciences. Significant contributions were provided by Kahneman et al. (1982) on the way actors frame their choices when facing uncertain events. Fischhoff et al. (in 1980) have significantly contributed to the identification of a set of explicative factors, or biases, between technical and none risk assessment. The reader can find more information on Kahneman and Tversky (1974), Sandman 
(1993) and Recchia (2001). These factors can roughly be grouped in a category called "individual cognitive or behavioral factors that influence the perception of risk problem". Examples can be the elimination of the cognitive dissonance that happens when new information, proven or not, is in contradiction with the values of an actor; in this situation, the information can be deliberately ignored. More examples listed in Merad (2010). In Douglas and Wildavsky (1982), Douglas (1986), Renn (1991a, b), Kasperson et al. (1987) and Walker et al. (1998) significant contributions are given on the "contextual such as cultural, collective and social factors that influence risk perception". These factors can be founded in Wright et al. (2006). Last but not least, the category of "organizational factors that can influence expertise in SSE". Many contributions can be found in Llory and et Montmayeul (2010), Dechy et al. (2011), (2012a, b), and Dien et al. (2012).

These biases can significantly impact the expertise process. As illustrations, let us for example point out the one case in nuclear domain, with the declaration of Jacques Repussard, in the journal Le Figaro, few months after the Fukushima disaster in 2011 that assumed an underestimation of the probability of major accidents of about $20^{11}$, and another one in safety cars engineering with the decision of production of the Ford Pinto during the 1970s (that became notorious for its tendency in rear-end collisions to leak fuel and explode into flames) that was considered as an only business decision and was based on a cost-benefit analysis badly and roughly done.

In this section, we wanted to avoid giving a jumbled list of the set B mainly, because we think that it is necessary to have an adapted list illustrated with examples according to the expertise organization experiences and SSE activity. Let us notice that explicating factors or biases that will influence the validity of expertise process is one strategy to avoid their inconvenience. Other strategies were proposed in the introduction section. Looking at safety measures, authors like Kervern (1994) or more recently Morel (2012) have tried to suggest strategies. Morel (2012) has proposed to increase systems safety by what he named meta-rules of high reliability and that he grouped in two distinct categories such as: collective meta-rules and cognitive meta-rules.

The step "issue situation" should take an end when both the DM and the Expert converge towards a common vision of the risk problematic (see Table 1).

The others steps suggested by Tsoukiàs (2007) such as the formulation of an issue, the choice of an assessment model, the framing of final recommendations can be transposed to the expertise process given the limitations point in the previous section.

\section{Conclusion}

We have argued in favor of a general integrated framework called an analytics and an ethics of expertise in the field of SSE, throughout the paper, based on our

\footnotetext{
${ }^{11}$ Accident nucléaire : «Il faut imaginer l'inimaginable ». Le Figaro17/06/2011. Actualité-Science. See: http://www.lefigaro.fr/sciences/2011/06/17/01008-20110617ARTFIG00610-accident-nucleaireilfaut-imaginer-1-inimaginable.php.
} 
experiences of risk problems. We have suggested that some guiding aspects, models and methods should be helpful to allow more validity and more legitimacy to the expertise process. These guiding aspects can have an explicative value to enlighten whether or not expertise process is robust and credible, but have not a causal validity even if they are based on experience feedback.

Aspects such as, on one hand, contextual and organizational conditions, and on the other hand cognitive and collective biases/factors, impact both the framing of risk problems and the framing of expertise conclusions and recommendations. Learning from failures of public expertises show that these aspects are neither fortuitous concomitances nor apparent pretexts for the generalization of rules on the validity and the legitimacy of expertise.

The thesis we have defended is that "expertise is a decision-aiding process for a final decision-maker but is a decision process by itself that needs a decision-aiding process". This paradox about a "helping process that needs help" shows that there is a need for framing both deontological and logical rules on the conduct and the governance of the public expertise process in SSE, but also a need for supporting the creative individual or collective process that expertise represents in practice. For this purpose, we have proposed in this paper a guiding process for expertise when facing risk problems based on experience feedback. This process aims to provide elements of guidance for experts/analyst to implement a decision-aiding process. A first theoretical and practical question, which is "how to guide the framing an "issue/problem" situation in SSE?" is so far partially solved. We have then pointed out a second issue that is: which kind of decision aiding should the Expert/Analyst provide to the DM or his representative considering the nature of risk problems in SSE? This question was partially answered by ensuring the respect of classical intrinsic properties of expertise recommendations and conclusions (e.g., effectiveness and robustness) and then by explicating and making explicit the contextual conditions.

Acknowledgments This research was funded by the French Ministry of Environment. The authors would like to thank Romuald Salmon and Pierre Toulhoat for their comments on earlier draft.

\section{References}

AFNOR (2003) NF X 50-110 Mai 2003-Qualité en expertise-Prescriptions générales de compétence pour une expertise

Allan G (2003) A critique of using grounded theory as a research method. Electron J Bus Res Methods 2(1):1-10

Al-Shemmeri T, Al-Kloub B, Pearman A (1997) Model choice in multicriteria decision aid. EJOR 97(3):550-560

Amendola A (2001) Recent paradigms for risk informed decision making. Saf Sci 40(2001):17-30

Assmuth T, Hilde M (2008) The significance of information frameworks in integrated risk assessment and management. Environ Sci Policy 11:71-86

Bouyssou D, Marchant Th, Perny P, Pirlot M, Tsoukiàs A, Vincke Ph (2000) Evaluation and decision models: a critical perspective. Kluwer, Dordrecht

Bouyssou D, Marchant Th, Pirlot M, Tsoukiàs A, Vincke Ph (2006) Evaluation and decision models: stepping stones for the analyst. Springer, Berlin

Chateauraynaud F, Torny D (1999) Les sombres précurseurs-une sociologie pragmatique des alertes et des crises. EHESS, Paris 
Cicolella A, Benoit-Browaey D (2005) Alertes santé—experts et citoyens face aux intérêts privés, édition Fayard

Cooke RM, Goossens LHJ (2000) Procedures guide for structured expert judgment. European commission report EUR 18820

David A (2001) Models implementation: a state of the art. Eur J Oper Res 134:459-480

Dechy N, Dien Y, Funnemark E, Roed-Larsen S, Stoop J, Valvisto T, Vetere A, Arellano L, on behalf of ESReDA's Accident Investigation Working Group (2012a) Results and lessons learned from the ESReDA's accident investigation working group: introducing article to "Safety Science" special issue on "Industrial Events Investigation". Safety Sci 50(6):1380-1391

Dechy N, Bourdeaux T, Ayrault N, Kordek M.-A, Le Coze J.-C (2004) First lessons of the Toulouse ammonium nitrate disaster, 21st September 2001, AZF Plant, France. J Hazard Mater 111 (special issue on JRC-ESReDA seminar on Safety accident investigation, Petten, the Netherlands, 12-13 May 2003)

Dechy N, Dien Y, Llory M (2010) For a culture of accidents devoted to industrial safety. In: $17^{\mathrm{e}}$ Congrès de Maîtrise des Risques et de Sûreté de Fonctionnement 5-7 octobre 2010 La Rochelle. http://www. imdr.fr

Dechy N, Rousseau J-M, Jeffroy F (2011) Learning lessons from accidents with a human and organizational factors perspective: deficiencies and failures of operating experience feedback systems. Eurosafe. Towards Convergence if technical nuclear safety practices in Europe. Nuclear safety: new challenges, gained experience and public expectations. Paris, 7 and 8 November 2011. 15 p. www.eurosafe-forum.org

Dechy N, Rousseau J-M, Llory M (2012b) Are organizational audits of safety that different from organizational investigation of accidents? In: Bérenguer C, Grall A, Guedes Soares C (eds) Advances in safety, reliability and risk management. Taylor \& Francis Group, London. pp 3009-3017, ISBN 978-0-415-68379-1

Dien Y, Dechy N, Guillaume E (2012) Accident investigation: from searching direct causes to finding indepth causes_-problem of analysis or/and of analyst? Safety Sci 50(6):1398-1407

Douglas M (1986) Risk acceptability according to social sciences. Routledge, Londre

Douglas M, Wildavsky A (1982) Risk and culture. An essay on the selection of technological and environmental dangers. University of California, Los Angeles

Epstein S (1994) Integration of cognitive and psychodynamic unconscious. Am Psychol 49:709-724

ESReDA Working Group on Accident Investigation Guideline for Safety Investigation of Accidents (2009) Guideline for safety investigation of accidents. www.esreda.org

Fairbrother A, Wenstel R, Sappington K, Wood W (2007) Frontier article. Framework for metals risk assessment. Ecotoxicol Environ Saf 68(2007):145-227

Favro K (2009) L'expertise : enjeux et pratiques. Editions La-voisier. Collection. Sciences du risque et du danger, $276 \mathrm{p}$

Georgopoulou E, Lalas D, Papagiannakis L (1997) A multicriteria decision aid approach for energy planning problems: the case of renewable energy option. EJOR 103(1):38-54

Gertman DI, Haney LN, Siub NO (1996) Representing context, cognition, and crew performance in a shutdown risk assessment. Reliab Eng Syst Saf 52:261-278

Goossens LHJ, Cooke RM, Hale AR, Rodić-Wiersma Lj (2008) Fifteen years of expert judgement at TUDelft. Saf Sci 46(2):234-244

Guitouni A, Martel J-M (1998) Tentative guidelines to help choosing an appropriate MCDA method. Eur J Ope Res 109(2):501-521

Horlick-Jones T (1998) Meaning and contextualization in risk assessment. Reliab Eng Syst Saf 59(1998):79-89

IRGC (2006) White paper on risk governance towards an integrative approach. http://www.irgc.org/IMG/ pdf/IRGC_WP_No_1_Risk_Governance_reprinted_version_pdf

Jacob L, Seillan H (2012) Dossier spécial «AZF ou SNPE ? La contre-expertise qui éclaire et explique ». No 124-juillet-août 2012-Editions Préventique

Jonas H (1985) The imperative of responsibility. In: Search of an ethics for the technological age. University of Chicago Press, Chicago, IL

Kahneman D, Lovallo D (1993) Timed choices and bold forecasts: a cognitive perspective on risk and risk taking. Manag Sci 39:17-31

Kahneman D, Tversky A (1974) Judgment under uncertainty: heuristics and bias. Sci 185:1124-1131

Kahneman D, Slovic P, Tversky A (1982) Judgment under uncertainty: heuristics and biases. Cambridge university press, Cambridge 
Kasperson R, Renn O, Slovic P (1987) The social amplification of risk: a conceptual framework. Risk Anal 8:177-187

Kelle U (2005) "Emergence" vs. "Forcing" of empirical data? A crucial problem of "Grounded Theory" reconsidered. Forum qualitative sozialforschung/forum: qualitative social research [On-line Journal] 6(2), Art. 27, paragraphs $49 \& 50$

Kervern G (1994) Latest advances in cindynics. Economica Editions. 112 pages, ISBN-13: 978-2717826944

Lagergren M (1998) What is the role and contribution of models to management and research in the health services? A view from Europe. Eur J Oper Res 105(1998):257-266

Landry M, Malouin JL, Oral M (1983) Model validation in operations research. Eur J Oper Res 14(3):207-220

Lannoy A, Procaccia H (1996) The EDF failure reporting system process, presentation and prospects. Reliab Eng Syst Saf 51(1996):147-158

Llory M (2000) The safety of risk-prone sociotechnical systems: engineers faced with ethical questions, In: Heriard B, Goujon P (eds) Technology and ethics. A European quest for responsible engineering, The European Ethics Network, France, pp 137-156

Llory M, et Montmayeul R (2010) L'accident et l'organisation, 176 pages. ISBN 978-2-911221-47-8. Editions : préventiques. Ouvrage coordonné par Llory M. et Montmayeul R. en collaboration avec Dechy N, Dien Y, Flori A, Fanchini H, et Merad M, Cru D, Fucks I, Voirin M

Martin PY, Turner B (1986) Grounded theory and organizational research. J Appl Behav Sci 22(2):141 (Retrieved June 21, 2009, from ABI/INFORM Global database. (Document ID: 1155984))

Merad M (2010) Aide à la décision et expertise en gestion des risques. Editions Lavoisier. 2010, 256 pages. ISBN : 978-2-7430-1265-6

Merad M, Dechy N (2010) Risk governance for sustainable territories: the French case and some challenges. Iinstitut de seguretat publica de catalunya (ISPC) Journal. http://www.raco.cat/index. php/RCSP/article/view/214968/285281

Merad M, Rodrigues N, Salvi O (2008) Urbanisation control around industrial Seveso sites: the French context. Int J Risk Assess Manag 8(1-2/2008):158-167

Merad M, Dechy N, Marcel F (2011) Adapting participative governance framework for the implementation of a sustainable development plan within an Organization. Ouvrage collectif coordonné par Karl HA, Flaxman M, Vargas-Moreno JC, Lynn Scarlett P Restoring and Sustaining Lands: Coordinating Science, Politics, and Action. Springer

Michaels D (2008) Doubt is their product: how industry's assault on science threatens your health. Oxford University Press. ISBN 0-19-530067-X

Mintzberg H (1989) Mintzberg on management: inside our strange world of organizations. Free Press/ Collier Macmillan, New York/London

Mishima A (1992) Bitter sea: the human cost of minamata disease. Kosei Publishing Company

Morel C (2012) Les décisions absurdes II-Comment les éviter. Gallimard Editions. 277 pages, ISBN 978-2-07-0135080

O'Keefe RM (1989) The evaluation of decision-aiding systems: guidelines and methods. Inf Manag 17(4):217-226

Oreskes N, Conway EM (2010) Merchants of doubt: how a handful of scientists obscured the truth on issues from tobacco smoke to global warming. Bloomsbury Press, New York

Ostanello A, Tsoukiàs A (1993) An explicative model of 'public' inter-organisational interactions'. Eur J Oper Res 70:67-82

Ostrom E (2005) Understanding institutional diversity. Princeton University Press, Princeton

Recchia V (2001) Risk communication and public perception of technological hazards

Reid SG (1999) Perception and communication of risk, and the importance of dependability. Struct Saf 21(1999):373-384

Renn O (1991) Risk communication and the social amplification of risk in Communicating risks to the public. Technology, risk, and society. Ouvrage collectif coordonné par Kasperson.R. Kluwer, Dordrecht

Renn O (1991) Risk communication and the social amplification of risk in Communicating risks to the public. Technology, risk, and society. Coordinated by Kasperson.R. Kluwer, Dordrecht

Renn O (1998) The role of risk perception for risk management. Reliab Eng Syst Saf 59(1998):49-62

Renn O (2008) Risk governance: coping with uncertainty in a complex world. Earthscan, London. Macmillan, London 
Rosqvist T (2010) Short communication-on the validation of risk analysis-a commentary. Reliab Eng Syst Saf 95(2010):1261-1265

Roy B (1985) Méthodologie Multicriteres d'Aide à la Décision. Economica, Paris 423 p

Roy B (1993) Decision science or decision-aid science? Eur J Oper Res 66:184-203

Sakamoto M, Murata K, Tsuruta K, Miyamoto K, Akagi H (2010) Retrospective study on temporal and regional variations of methylmercury concentrations in preserved umbilical cords collected from inhabitants of the Minamata area. Ecotoxicol Environ Saf 73(6):1144-1149

Sandman P (1993) Responding to community outrage: strategies for effective risk communication. Edition: American Industrial Hygiene Association

Slovic P, Fischoff B (1977) On the psychology of experimental surprises. J Exp Psychol 3:544-551

Slovic P, Fischoff B, Lichtenstein S (1980) Facts and fears: understanding perceived risks. Society of Risk Assessments: How safe is Safe enough? Plenum Press, New York

Slovic P, Finucane M, Peters E, Mac Gregor D (2002) Rational actors or rational fools: implications of the affect heuristic for behavioral economics. J Socio-Economics 31:329-342

Stanovich KE, West RF (2000) Individual differences in reasoning: implications for the rationality debate. Behav Brain Sci 23:645-726

Thomas G, James D (2006) Re-inventing grounded theory: some questions about theory, ground and discovery. Br Educ Res J 32(6):767-795

Tsoukiàs A (2007) On the concept of decision aiding process: an operational perspective. Annals of Operational Research. Springer Science + Business Media

Tsoukiàs A (2008) From decision theory to decision aiding methodology. Eur J Oper Res 187:138-161

Vaughan D (1996) The challenger launch decision: risky technology, culture, and deviance at Nasa. The University of Chicago Press, Chicago

Vincke P (1986) Analysis of multicriteria decision aid in Europe. EJOR 25(2):160-168

Walker G, Simms P, Wynne B, Irwin A (1998) Public perception of risks associated with major accidental hazards. HSE

Wisner A (1982) La psychologie de l'homme au travail ou la psychologie n'est pas une technique. Psychologie française 27(2):107-112

Wisner A (1995) Understanding problem building: ergonomic work analysis, an analytical approach. Ergonomic 38:596-606

Wright D, Dressel K, Dien Y, Merad M (2006) Delivrable 1-the dimensions of risk communication. STRAC "STAkeholders in Risk Communications". FP6-2003-SCIENCE-AND-SOCIETY-7 (online) 